\title{
BMJ Open Epidemiology of chronic pain in children and adolescents: a protocol for a systematic review update
}

\author{
Perri R Tutelman (ID ,1,2 Charlotte L Langley (D) ,2 Christine T Chambers (i) ,1,2,3 \\ Jennifer A Parker (1) , ${ }^{2}$ G Allen Finley (1) ,1,2,4 Darlene Chapman, ${ }^{5}$ Gareth T Jones, ${ }^{6}$ \\ Gary J Macfarlane (1) , ${ }^{6}$ Justina Marianayagam ${ }^{7,8}$
}

To cite: Tutelman PR, Langley CL, Chambers CT, et al. Epidemiology of chronic pain in children and adolescents: a protocol for a systematic review update. BMJ Open 2021;11:e043675. doi:10.1136/ bmjopen-2020-043675

- Prepublication history and supplemental material for this paper is available online. To view these files, please visit the journal online (http://dx.doi org/10.1136/bmjopen-2020043675).

Received 11 August 2020 Revised 28 December 2020 Accepted 18 January 2021

Check for updates

(C) Author(s) (or their employer(s)) 2021. Re-use permitted under CC BY-NC. No commercial re-use. See rights and permissions. Published by BMJ.

For numbered affiliations see end of article.

Correspondence to Dr Christine T Chambers; Christine.Chambers@dal.ca

\section{ABSTRACT}

Introduction Chronic pain, defined as persistent or recurring pain or pain lasting longer than 3 months, is a common childhood problem and can profoundly impact children's physical, psychological and social functioning. The last comprehensive systematic review estimating the prevalence of chronic pain in children and adolescents was published in 2011. Since then, the literature on paediatric chronic pain has grown substantially. This manuscript outlines a protocol for an updated systematic review to provide updated estimates of the prevalence of various forms of chronic pain in children and adolescence. The review will also examine the relationship between sociodemographic and psychosocial factors related to chronic pain prevalence.

Methods and analysis This review will follow Preferred Reporting Items for Systematic Reviews and Meta-Analyses guidelines. We will search EMBASE, PubMed, CINAHL and PsycINFO for observational studies published in English between 2009 and 2020 reporting population-based estimates of chronic non-disease-related pain prevalence in children or adolescents (age $\leq 19$ years). Two independent reviewers will screen the titles and abstracts retrieved from the search based on predefined eligibility criteria. The full texts of relevant studies will then be assessed by two reviewers. Studies meeting inclusion criteria will be categorised according to the type of pain investigated: headache only, abdominal pain only, back pain only, musculoskeletal pain, combined pain, general pain and other pain. Data will be extracted using customised forms and studies will be assessed for risk of bias using a 10-item tool developed by Hoy et al (2012). A narrative synthesis will summarise the prevalence estimates of paediatric chronic pain and associated sociodemographic and psychosocial correlates. Meta-analyses and meta-regressions will be performed if the data permit.

Ethics and dissemination Ethical approval is not required.

Findings will be disseminated through publication in an academic journal, presentations at conferences and in various media.

PROSPERO registration number CRD42020198690.

\section{INTRODUCTION}

Chronic pain, often defined as persistent or recurring pain or pain lasting longer than 3 months, ${ }^{1}$ is a common problem in childhood. Although numerous recent population-based studies have provided estimates of the prevalence of chronic
Strengths and limitations of this study

- This systematic review will provide updated estimates of the prevalence of various forms of chronic pain in children and adolescents.

- A comprehensive literature search will be conducted to identify eligible studies.

- This systematic review will follow the Preferred Reporting Items for Systematic Reviews and MetaAnalyses guidelines and will assess included articles for risk of bias using a validated quality assessment tool.

- Heterogeneity in study methods and populations may limit our ability to pool the findings across studies.

- The findings of this review will enhance our understanding of the current burden of paediatric chronic pain which may help inform the treatment and allocation of clinical resources for this population.

pain in children and adolescents, the reported proportions have varied considerably across studies. For example, the prevalence of primary headache disorders in children and adolescents in recent population-based studies has ranged from $19.4 \%$ to $66.4 \%,{ }^{23}$ while estimates of the prevalence of functional gastrointestinal disorders have varied from $4.6 \%$ to $31.2 \% .{ }^{45}$ Due to the wide variability in the reported estimates, the current epidemiology of chronic pain in children and adolescents is unclear. The uncertainty regarding the number of children and adolescents impacted by chronic pain may limit the appropriate allocation of clinical services for this population, which are crucial given the pervasive and long-term consequences of chronic pain on young people.

Chronic pain can have a profound impact on children and adolescents. Children who experience chronic pain are at increased risk for depression and anxiety, ${ }^{67}$ school absences, ${ }^{8}$ social isolation ${ }^{9}$ and poorer quality of life. ${ }^{10}$ Approximately $5 \%$ of children with chronic pain experience severe levels of pain which significantly impact their daily functioning. ${ }^{11}$ Unfortunately, 
many children with chronic pain become adults with chronic pain. In a prospective study of paediatric patients with functional abdominal pain, 35\% continued to report recurrent abdominal symptoms when reassessed in adulthood. ${ }^{12}$ Similarly, in 14-year follow-up study of adolescents with frequent headaches, $19 \%$ continued to report weekly headaches in young adulthood. ${ }^{13}$ These rates are similar to retrospective reports of chronic pain in childhood by adults with chronic pain. In a study of adult patients with chronic pain, $17 \%$ of participants reported their pain originated in childhood or adolescence. $^{14}$

The persistence of chronic pain from childhood to adulthood has significant social and economic consequences. For instance, children with chronic pain are at risk for opioid misuse ${ }^{15}$ and psychiatric morbidity in adulthood, ${ }^{16}$ and paediatric chronic pain is associated with high rates of outpatient appointments, emergency department visits and hospitalisations, all resulting in increased healthcare costs. ${ }^{17-19}$ The total annual cost of paediatric chronic pain in the USA is estimated to be $\$ 19.5$ billion. ${ }^{18}$ The significant individual, social and economic burden of paediatric chronic pain, and its persistence into adulthood, demonstrates the importance of understanding the epidemiology of this disease in order to improve treatment and reduce the impact that chronic pain has on the lives of children and adolescents.

The most recent comprehensive review on the epidemiology of chronic pain in children and adolescents was published in $2011 .^{20}$ This review estimated that the median prevalence of chronic pain in children and adolescents ranged from $11 \%$ to $38 \%$ depending on pain type and varied substantially across studies. The prevalence of chronic pain was found to be higher in girls and prevalence proportions increased with age. ${ }^{20}$ The review identified several psychosocial correlates of chronic pain in children and adolescents such as lower socioeconomic status, anxiety, depression and low self-esteem. ${ }^{20}$ However, other correlates of chronic pain in children and adolescents, such as sleep ${ }^{2122}$ and posttraumatic stress disorder, ${ }^{2324}$ have since been identified and were not consistently examined at the population level at the time of the past review. The review also identified several gaps in the understanding of the epidemiology of chronic pain in children and adolescents, including restricted age ranges and lack of longitudinal studies. ${ }^{20}$ Furthermore, at the time of the past review the quality of included studies was generally low to moderate and methodological limitations, such as inconsistent definitions of pain between studies, made it difficult to estimate overall prevalence proportions. ${ }^{20}$

The literature on paediatric pain is growing exponentially; a recent bibliometric analysis revealed that there was nearly a 40 -fold increase in the number of publications on paediatric pain from 1975 to $2010 .^{25}$ Since the last comprehensive systematic review on the epidemiology of chronic pain in children and adolescents, ${ }^{20}$ numerous populationbased studies estimating the prevalence of various forms of paediatric chronic pain have been published. ${ }^{26-28} \mathrm{~A}$ few recent reviews estimating the prevalence of certain forms of chronic pain, such as functional abdominal pain ${ }^{29}$ and headache, ${ }^{30} 31$ have also been conducted. However, in order to appropriately meet the clinical needs of children and adolescents with chronic pain, an updated review that estimates the proportion of various forms of chronic pain and examines key sociodemographic and psychosocial correlates of chronic pain in children and adolescents is needed.

This manuscript outlines a protocol for a systematic review to update a prior review synthesising the published literature on the prevalence of chronic pain in children and adolescents. Specifically, the objectives of this review are to: (1) provide updated estimates of the prevalence of various forms of chronic pain (headache, abdominal pain, back pain, musculoskeletal pain, combined pain, general pain and other pain) in children and adolescents; (2) provide an updated examination of sociodemographic (eg, age, sex, race) and psychosocial (eg, anxiety, depression, sleep) factors related to the prevalence of chronic pain in children and adolescents; and (3) assess study quality and identify gaps in the literature and areas for future research.

\section{METHODS AND ANALYSIS}

This systematic review protocol was prepared in accordance with the Preferred Reporting Items for Systematic Reviews and Meta-Analyses Protocols (PRISMA-P) guidelines (see online supplemental file 1). ${ }^{32}$ The study protocol was registered with the International Prospective Register of Systematic Reviews (PROSPERO) on 3 September 2020. In the event of an amendment to this protocol, the date of the amendment, a description of the change and the rationale will be documented and recorded in PROSPERO. The dates for this review are 14 May 2020-1 April 2021 (expected).

\section{Search strategy}

We will search the following electronic databases: EMBASE, PubMed, CINAHL and PsycINFO. The search terms will be comprised of three conceptual blocks: (1) pain terms (eg, musculoskeletal pain, back pain, headache, abdominal pain, recurrent pain); (2) paediatric terms (eg, child, adolescent, boy, girl) and (3) epidemiological terms (eg, epidemiology, prevalence, frequency). The searches will be restricted to English-language articles, human studies, and manuscripts published between January 2009 and June 2020 (the original systematic review ${ }^{20}$ included studies published up until 2009).

\section{Eligibility criteria}

Studies will be eligible for inclusion if they meet the following criteria:

1. Observational studies using a population-based sampling frame to estimate the prevalence of chronic pain in children or adolescents (study sample age $\leq 19$ years).

2. Studies examining the prevalence of chronic pain in children and adolescents, defined as pain with a minimum duration of at least 3 months or pain that is described as chronic, persistent or recurrent. This definition was selected to align with current conceptualisations of chronic pain ${ }^{1}$ while allowing for flexibility to accommodate established diagnostic criteria for common childhood chronic pain conditions (eg, functional abdominal pain ${ }^{33}$ and migraine). ${ }^{34}$ 
3. Studies published in peer-reviewed journals in English.

Studies meeting the following criteria will be excluded:

1. Studies with sampling frames not deemed to be population based.

2. Case studies, conference abstracts, dissertations, reviews, book chapters and qualitative studies.

3. Studies reporting on the prevalence of chronic pain in adults (sample age is exclusively $\geq 20$ years).

4. Studies of non-human samples.

5. Studies examining the prevalence of chronic pain in specific subpopulations, such as children and adolescents with chronic illnesses (eg, cancer, arthritis) or other health conditions (eg, cerebral palsy, muscular dystrophy).

Studies reporting on multiple populations (eg, adults and children), where data on one or more subpopulations that fit the eligibility criteria for this review can be separately identified, will be included and the relevant data will be extracted.

\section{Screening and data extraction}

Literature search results will be transferred to Covidence systematic review management software and duplicates will be removed. An initial title/abstract review of studies retrieved by the search will be independently conducted by two members of the study team to determine which studies potentially met the inclusion/exclusion criteria. Articles included from the title/abstract review phase will then be reviewed in full by two reviewers. The two reviewers will be blinded to each other's decisions. Discrepancies regarding the eligibility of a study will be resolved by consensus, and if necessary, through discussion with a third reviewer.

Data will be extracted from included studies using customised forms. Extracted information will include study design, location where the study was conducted, number of participants, participant demographics (eg, age, sex, race), study sample (eg, headache only, abdominal pain only, back pain only, musculoskeletal pain, combined pain, general pain and other pain), study methodology, prevalence proportion of chronic pain, and sociodemographic (eg, age, sex, race, parent education, household income) and psychosocial (eg, anxiety, depression, sleep, post-traumatic stress) predictors of chronic pain. In accordance with the PRISMA guidelines, ${ }^{35}$ the number of studies meeting inclusion criteria will be recorded and the reason for exclusion of those not included will be documented.

\section{Quality assessment}

Two independent reviewers will assess study quality using the 10-item tool developed by Hoy et al. ${ }^{36}$ This tool was developed to assess external and internal validity of prevalence studies. ${ }^{36}$ Response options for each item are either 'yes' (indicating low risk of bias) or 'no' (indicating high risk of bias). ${ }^{36}$ The tool will be adapted for this review if necessary. Consensus will be reached by discussion between the reviewers.

\section{Data synthesis}

A narrative synthesis will summarise the prevalence proportions of chronic pain in children and adolescents in the following categories: headache only; abdominal pain only; back pain only; musculoskeletal pain; combined pain; general pain and other pain. Additionally, the relationship between psychosocial factors (eg, sleep, anxiety and depression) and sociodemographic factors (eg, sex, age, race and indicators of socioeconomic status such as, but not limited to, household income, parental level of education and urban vs rural area of residence), and chronic pain in children and adolescents will be reviewed.

Depending on the heterogeneity of included studies, the prevalence proportions of chronic pain will be calculated using median prevalence proportions and/or metaanalysis. When at least two or more studies are comparable in terms of the study sample (eg, category of chronic pain) and methodology (eg, operationalisation of chronic pain), we will pool the effects to determine the prevalence proportion of chronic pain across studies. Similarly, if studies have used similar methods to examine the relationship between certain sociodemographic and/or psychosocial variables in comparable chronic pain samples, we will conduct separate meta-regressions to examine the relationship between these variables and chronic pain across studies.

Reporting of this systematic review will follow PRISMA guidelines. ${ }^{35}$ A PRISMA flow diagram will be included in the final manuscript of this review.

\section{Ethics and dissemination}

Ethical approval will not be sought for this study, as no human subject participants will be involved. A manuscript outlining the results of this review will be submitted for publication in a peer-reviewed academic journal and for presentation at relevant academic conferences. Results will be publicly disseminated through social media, news and media outlets, and newsletters and blog posts, as appropriate.

\section{IMPLICATIONS OF THE REVIEW}

We anticipate that the results from this review will enhance our understanding of the current burden of paediatric chronic pain which may help inform treatment and allocation of clinical resources for this population. Furthermore, findings from this study will identify priority areas for research on the epidemiology of chronic pain to guide future research efforts. Through our planned knowledge translation efforts, findings of the review will be disseminated not only to clinicians and scientists, but also to patients and families, which may aid in public awareness and advocacy efforts.

\section{PATIENT AND PUBLIC INVOLVEMENT}

This protocol was designed in collaboration with a patient partner and coauthor, JM. JM will remain involved as a patient partner throughout all steps of the review.

\section{Author affiliations}

${ }^{1}$ Department of Psychology and Neuroscience, Dalhousie University, Halifax, Nova Scotia, Canada

${ }^{2}$ Centre for Pediatric Pain Research, IWK Health Centre, Halifax, Nova Scotia, Canada

${ }^{3}$ Department of Pediatrics, Dalhousie University, Halifax, Nova Scotia, Canada ${ }^{4}$ Department of Anesthesia, Pain Management and Perioperative Medicine, Dalhousie University, Halifax, Nova Scotia, Canada

${ }^{5}$ Library Services, IWK Health Centre, Halifax, Nova Scotia, Canada 
${ }^{6}$ Epidemiology Group and Aberdeen Centre for Arthritis and Musculoskeletal Health, University of Aberdeen, Aberdeen, UK

${ }^{7}$ Patient Partner, Thunder Bay, Ontario, Canada

${ }^{8}$ Northern Ontario School of Medicine, Thunder Bay, Ontario, Canada

Twitter Perri R Tutelman @PerriTutelman, Christine T Chambers @DrCChambers, Jennifer A Parker @JAParkerPhD, Gareth T Jones @hteraG_senoJ and Gary J Macfarlane @aberdeenepi

Contributors CTC conceived the study idea. CLL, PRT and JAP designed the study protocol, data extraction and statistical analysis, and wrote the first draft of the manuscript. CTC, DC, GAF, GTJ, GJM and JM provided critical insights at all stages. All authors approved and contributed to the final manuscript.

Funding This work was supported by an operating grant from the Canadian Institutes of Health Research (FRN167902) awarded to CTC and funding from the Dalhousie Medical Research Foundation (DMRF). CTC is the senior author and is supported by a Tier 1 Canada Research Chair with infrastructure support from the Canada Foundation for Innovation. CLL is supported by an IWK Health Centre Summer Studentship (1025420). PRT is supported by a Research Nova Scotia Scholars Award, a Nova Scotia Graduate Scholarship and an IWK Graduate Studentship Award, and is a trainee member of Pain Child Health (PICH).

Competing interests None declared.

Patient consent for publication Not required.

Provenance and peer review Not commissioned; externally peer reviewed.

Supplemental material This content has been supplied by the author(s). It has not been vetted by BMJ Publishing Group Limited (BMJ) and may not have been peer-reviewed. Any opinions or recommendations discussed are solely those of the author(s) and are not endorsed by BMJ. BMJ disclaims all liability and responsibility arising from any reliance placed on the content. Where the content includes any translated material, BMJ does not warrant the accuracy and reliability of the translations (including but not limited to local regulations, clinical guidelines, terminology, drug names and drug dosages), and is not responsible for any error and/or omissions arising from translation and adaptation or otherwise.

Open access This is an open access article distributed in accordance with the Creative Commons Attribution Non Commercial (CC BY-NC 4.0) license, which permits others to distribute, remix, adapt, build upon this work non-commercially, and license their derivative works on different terms, provided the original work is properly cited, appropriate credit is given, any changes made indicated, and the use is non-commercial. See: http://creativecommons.org/licenses/by-nc/4.0/.

\section{ORCID iDs}

Perri R Tutelman http://orcid.org/0000-0001-6013-7402

Charlotte L Langley http://orcid.org/0000-0002-0291-8734

Christine T Chambers http://orcid.org/0000-0002-7138-916X

Jennifer A Parker http://orcid.org/0000-0001-9900-4703

G Allen Finley http://orcid.org/0000-0003-4579-7749

Gary J Macfarlane http://orcid.org/0000-0003-2322-3314

\section{REFERENCES}

1 Treede R-D, Rief W, Barke A, et al. A classification of chronic pain for ICD-11. Pain 2015;156:1003-7.

2 Al-Hashel JY, Ahmed SF, Alroughani R. Prevalence and burden of primary headache disorders in Kuwaiti children and adolescents: a community based study. Front Neurol 2019;10:793.

3 Malik AH, Shah PA, Yaseen Y. Prevalence of primary headache disorders in school-going children in Kashmir Valley (north-west India). Ann Indian Acad Neurol 2012;15:100-3.

4 Zheng S, Fu W, Zhou J, et al. Prevalence and related factors of irritable bowel syndrome among middle-school students in areas affected by Wenchuan earthquake: an epidemiological study. J Clin Gastroenterol 2012;46:345-6.

5 Demirceken FG, Kurt G, Dulkadir R. Functional dyspepsia in children: a Turkish prospective survey in Kirikkale Province, 2010.

6 Shelby GD, Shirkey KC, Sherman AL, et al. Functional abdominal pain in childhood and long-term vulnerability to anxiety disorders. Pediatrics 2013;132:475-82

7 Simons LE, Sieberg CB, Claar RL. Anxiety and impairment in a large sample of children and adolescents with chronic pain. Pain Res Manag 2012:17:93-7.

8 Logan DE, Gray LS, Iversen CN, et al. School Self-Concept in adolescents with chronic pain. J Pediatr Psychol 2017;42:892-901.
9 Maes M, Van den Noortgate W, Fustolo-Gunnink SF, et al. Loneliness in children and adolescents with chronic physical conditions: a metaanalysis. J Pediatr Psychol 2017;42:622-35.

10 Gold JI, Yetwin AK, Mahrer NE, et al. Pediatric chronic pain and healthrelated quality of life. J Pediatr Nurs 2009;24:141-50.

11 Huguet A, Miró J. The severity of chronic pediatric pain: an epidemiological study. J Pain 2008;9:226-36.

12 Walker LS, Dengler-Crish CM, Rippel S, et al. Functional abdominal pain in childhood and adolescence increases risk for chronic pain in adulthood. Pain 2010;150:568-72.

13 Larsson B, Sigurdson JF, Sund AM. Long-Term follow-up of a community sample of adolescents with frequent headaches. $J$ Headache Pain 2018;19:79.

14 Hassett AL, Hilliard PE, Goesling J, et al. Reports of chronic pain in childhood and adolescence among patients at a tertiary care pain clinic. J Pain 2013;14:1390-7.

15 Groenewald CB, Law EF, Fisher E, et al. Associations between adolescent chronic pain and prescription opioid misuse in adulthood. $J$ Pain 2019;20:28-37.

16 Noel M, Groenewald CB, Beals-Erickson SE, et al. Chronic pain in adolescence and internalizing mental health disorders: a nationally representative study. Pain 2016;157:1333-8.

17 Ho IK, Goldschneider KR, Kashikar-Zuck S, et al. Healthcare utilization and indirect burden among families of pediatric patients with chronic pain. J Musculoskelet Pain 2008;16:155-64.

18 Groenewald CB, Essner BS, Wright D, et al. The economic costs of chronic pain among a cohort of treatment-seeking adolescents in the United States. J Pain 2014;15:925-33.

19 Tumin D, Drees D, Miller R, et al. Health care utilization and costs associated with pediatric chronic pain. J Pain 2018;19:973-82.

20 King S, Chambers CT, Huguet A, et al. The epidemiology of chronic pain in children and adolescents revisited: a systematic review. Pain 2011;152:2729-38.

21 Evans S, Djilas V, Seidman LC, et al. Sleep quality, affect, pain, and disability in children with chronic pain: is affect a mediator or Moderator? J Pain 2017;18:1087-95.

22 Murphy LK, Palermo TM, Tham SW, et al. Comorbid sleep disturbance in adolescents with functional abdominal pain. Behav Sleep Med 2020;0:1-10.

23 Noel M, Wilson AC, Holley AL, et al. Posttraumatic stress disorder symptoms in youth with vs without chronic pain. Pain 2016;157:2277-84.

24 Stahlschmidt L, Rosenkranz F, Dobe M, et al. Posttraumatic stress disorder in children and adolescents with chronic pain. Health Psychol 2020;39:463-70.

25 Caes L, Boerner KE, Chambers CT, et al. A comprehensive categorical and bibliometric analysis of published research articles on pediatric pain from 1975 to 2010. Pain 2016;157:302-13.

26 Oswari H, Alatas FS, Hegar B, et al. Functional abdominal pain disorders in adolescents in Indonesia and their association with family related stress. BMC Pediatr 2019;19:342-5.

27 Aartun E, Hartvigsen J, Wedderkopp N, et al. Spinal pain in adolescents: prevalence, incidence, and course: a school-based two-year prospective cohort study in 1,300 Danes aged 11-13. BMC Musculoskelet Disord 2014:15:187.

28 Arruda MA, Arruda R, Guidetti V, et al. Psychosocial adjustment of children with migraine and tension-type headache - a nationwide study. Headache 2015;55 Suppl 1:39-50.

29 Korterink JJ, Diederen K, Benninga MA, et al. Epidemiology of pediatric functional abdominal pain disorders: a meta-analysis. PLoS One 2015;10:e0126982.

30 Albers L, von Kries R, Heinen F, et al. Headache in school children: is the prevalence increasing? Curr Pain Headache Rep 2015;19:4.

31 Wöber-Bingöl C. Epidemiology of migraine and headache in children and adolescents topical collection on childhood and adolescent headache. Curr Pain Headache Rep 2013;17:1-11.

32 Moher D, Shamseer L, Clarke M. Preferred reporting items for systematic review and meta-analysis protocols (PRISMA-P) 2015 statement. Rev Espanola Nutr Humana Diet 2016;20:148-60.

33 Hyams JS, Di Lorenzo C, Saps M, et al. Childhood functional gastrointestinal disorders: Child/Adolescent. Gastroenterology 2016;150:1456-68.

34 Headache Classification Committee of the International Headache Society (IHS). The International classification of headache disorders. . 3rd ed. Cephalalgia, 2018: 38. 1-211.

35 Moher D, Liberati A, Tetzlaff J, et al. Preferred reporting items for systematic reviews and meta-analyses: the PRISMA statement. PLoS Med 2009;6:e1000097.

36 Hoy D, Brooks $\mathrm{P}$, Woolf $\mathrm{A}$, et al. Assessing risk of bias in prevalence studies: modification of an existing tool and evidence of interrater agreement. J Clin Epidemiol 2012;65:934-9. 